\title{
Efficacy of 1/3-Dose Verteporfin Photodynamic Therapy for Subacute Central Serous Chorioretinopathy
}

\author{
Li Xu*, Qiushi Liu, Xiaotong Zhuang, Dongning Liu \\ Department of Ophthalmology, The Fourth People’s Hospital of Shenyang, Shenyang, China \\ Email: *xu-li1149@163.com
}

How to cite this paper: Xu, L., Liu, Q.S., Zhuang, X.T. and Liu, D.N. (2017) Efficacy of 1/3-Dose Verteporfin Photodynamic Therapy for Subacute Central Serous Chorioretinopathy. Open Journal of Ophthalmology, 7, 310-318.

https://doi.org/10.4236/ojoph.2017.74040

Received: October 1, 2017

Accepted: November 14, 2017

Published: November 17, 2017

Copyright (c) 2017 by authors and Scientific Research Publishing Inc. This work is licensed under the Creative Commons Attribution International License (CC BY 4.0).

http://creativecommons.org/licenses/by/4.0/

\begin{abstract}
Purpose: To study the safety and efficacy of $1 / 3$-dose verteporfin photodynamic therapy (PDT) for subacute central serous chorioretinopathy (CSC). Methods: In this case series, 59 eyes (59 patients) diagnosed with subacute CSC in Shenyang the $4^{\text {th }}$ hospital from January 2014 to December 2015 were treated with 1/3-dose verteporfin PDT and followed up for at least 1 year. The symptoms and the diagnosed history were more than 3 months but shorter than 6 months. The central foveal thickness (CFT), neuroretinal thickness (NRT), height of subfoveal retinal fluid (SRF), and subfoveal choroidal thickness (SCT) were observed at baseline and after treated at 1, 2, 3, 6 and 12 months with EDI-OCT, Best-corrected visual acuity ( BCVA) was also studied at the same time. Results: After 1, 2, 3 and 6 months of 1/3-dose verteporfin $\mathrm{PDT}$ treatment, the BCVA improved significantly $(\mathrm{P}<0.01)$ comparing to the baseline. The LogMAR BCVA of baseline and 1, 2, 3, 6 and 12 months was $0.553 \pm 0.11,0.201 \pm 0.13,0.149 \pm 0.10,0.137 \pm 0.09,0.143 \pm 0.07$ and $0.148 \pm$ 0.08 respectively. OCT scan showed that the subfoveal choroid thickness (SCT) was $458.25 \pm 24.23 \mu \mathrm{m}, 379.31 \pm 9.31 \mu \mathrm{m}, 353.24 \pm 8.57 \mu \mathrm{m}, 349.35 \pm$ $7.51 \mu \mathrm{m}, 365.23 \pm 8.08 \mu \mathrm{m}$ and $358.41 \pm 7.96$ at baseline and 1, 2, 3, 6 and 12 months respectively. The SCT of 1,2, and 3 months after treatment reduced significantly $(\mathrm{P}<0.01)$ comparing to baseline. The neuroretinal thickness (NRT) was not significantly different after $1 / 3$-dose PDT $(\mathrm{P}>0.05)$. The height of SRF changed significantly. There was no retinal pigment epithelium atrophy and choroidal neovascularization (CNV) in all cases after more than 12 months follow-up. Conclusion: Treatment of 1/3 dose verteporfin PDT could safely and effectively reduce expansion of choroidal vessel and choroidal choriocapillary, promoting absorbance of subretinal fluid for subacute CSC. 1/3-dose verteporfin PDT may be an alternative method to treat the subacute CSC.
\end{abstract}




\section{Keywords}

Subacute Central Serous Chorioretinopathy (CSC), 1/3-Dose Verteporfin Photodynamic Therapy (PDT), Subfoveal Choroidal Thickness (SCT), Neuroretinal Thickness (NRT), Central Foveal Thickness (CFT)

\section{Introduction}

Central serous chorioretinopathy (CSC) frequently affects young-to-middle-aged men [1] [2]. The exact etiology of CSC is unknown, although disturbances in choroidal circulation are suggested to be related to CSC. EDI-OCT shows the characteristics of the accumulation of subretinal fluid (SRF). Indocyanine green angiography (ICGA) has shown the hyperpermeability of choroidal vessels in eyes with CSC [3] [4]. CSC is generally recognized as a self-limiting disease with good prognosis, and resolves spontaneously within 3 - 4 months [3] [4]. However, in the long term, approximately half of the patients experience persistent or recurrent SRF [5] [6]. In these patients, the prognosis can be poor due to complications such as diffuse atrophy of the retinal pigment epithelium (RPE), subretinal fibrosis, and thinning of the outer sensory retina [3] [7].

In 2003, photodynamic therapy (PDT) with full-dose verteporfin was reported as a new treatment for chronic CSC [8]. However, complication such as secondary choroidal neovascularization (CNV), pigmentary changes of the RPE, and persistent choroidal ischemia can occur after full-dose verteporfin PDT [8] [9] [10] [11]. In recent years, PDT with half-dose verteporfin has been conducted in chronic CSC with similar effects and reduced complications [12] [13] [14] [15] [16]. Although the dose verteporfin is half, there are also some complications such as the CNV, atrophy of RPE [3] [17]. There are some reports showing that 1/3-dose verteporfin PDT was safe and effective in chronic CSC [18]. The purpose of this study was to determine the safety and efficacy of 1/3-dose verteporfin PDT in subacute CSC. We investigated the results of the LogMAR BCVA after 1/3-dose verteporfin PDT at deferent time. The choroidal thickness and neuroretinal thickness had also been assessed. The subacute CSC was diagnosed that symptoms were more than 3 months but shorter than 6 months. The timing of treatment was also concerned.

\section{Methods}

1) Study Participants

We reviewed the results and the data of subacute CSC patients who have received 1/3-dose verteporfin PDT from Jan 2014 to Des 2015 at Department of Ophthalmology, Shenyang the forth people hospital. CSC was diagnosed as detachment of the neurosensory retina from the macular caused by idiopathic leakage or diffuse leakage from the RPE. Leakage from RPE was detected by fluorescein angiography (FA) and choroidal vascular hyperpermeability was de- 
tected by ICGA. Subacute CSC was diagnosed when symptoms more than 3 months but shorter than 6 months and the SRF persisted for shorter than 6 months. The inclusion and exclusion criteria are listed in Table 1. This study was approved by the Institutional Review Board of the forth people hospital. Written informed consent was obtained from all patients. The procedures of this study followed the tenets of the Declaration of Helsinki.

2) All patients received $1 / 3$ of the standard dose of verteporfin $\left(1.8 \mathrm{mg} / \mathrm{m}^{2}\right.$ PDT), which was infused over $10 \mathrm{~min}$, followed by laser treatment $15 \mathrm{~min}$ after. The laser delivered to cover the area of choroidal hyperpermeability that was detected during the middle phase of ICG. The total light energy was $50 \mathrm{~J} / \mathrm{cm}^{2}$. The power was $600 \mathrm{~mW} / \mathrm{cm}^{2}$. After treatment, patients were instructed to avoid sunlight for 3 days.

3) Baseline and follow-up examination

Patients were assessed at baseline and at 1,2, 3, 6 and 12 months after PDT. Measurements of best-corrected visual acuity (BCVA), slit-lamp examination, dilated fundus examination, and OCT were performed at each visit. BCVA was measured based on the Chinese standard decimal visual acuity chart at $5 \mathrm{~m}$ and was converted to the logarithm of the minimum angle of resolution (LogMAR) scale for analysis. OCT was performed with enhanced depth imaging (EDI-OCT)

Table 1. Inclusion and exclusion criteria of the patients recruited in the study.

\begin{tabular}{|c|c|}
\hline \multirow{5}{*}{$\begin{array}{l}\text { Inclusion } \\
\text { criteria }\end{array}$} & $\begin{array}{l}\text { 1) Subjective symptoms or persistence of subfoveal fluid on OCT for more than } 3 \\
\text { months but shorter than } 6 \text { months }\end{array}$ \\
\hline & $\begin{array}{l}\text { 2) Patients whose follow-up period was available for more than } 12 \text { months after } \\
\text { 1/3-dose verteporfin PDT }\end{array}$ \\
\hline & 3) Presence on FA of active angiographic leakage secondary to CSC \\
\hline & $\begin{array}{l}\text { 4) Choroidal vascular hyperpermeability and abnormal dilation of choroidal } \\
\text { vasculature, consistent with a diagnosis of CSC }\end{array}$ \\
\hline & $\begin{array}{l}\text { 5) Difficult to treat by Laser photocoagulation (LP) because of leakage from the } \\
\text { subfoveal, parafoveal, or broad area }\end{array}$ \\
\hline \multirow{8}{*}{$\begin{array}{l}\text { Exclusion } \\
\text { criteria }\end{array}$} & 1) Auto fluorescence shows some atrophy RPE \\
\hline & 2) Symptoms more than 6 months \\
\hline & $\begin{array}{l}\text { 3) Presence of CNV or other maculopathy or other ocular diseases that could } \\
\text { affect visual acuity }\end{array}$ \\
\hline & 4) Presence of choroidopathy that may affect choroidal thickness \\
\hline & $\begin{array}{l}\text { 5) No history of PDT, LP, anti VEGF injections, TA injections or other treatments } \\
\text { within } 3 \text { months before } 1 / 3 \text {-dose PDT for CSC }\end{array}$ \\
\hline & $\begin{array}{l}\text { 6) Optic media opacity that could interfere with adequate acquisition of OCT, FA, } \\
\text { and ICG images }\end{array}$ \\
\hline & 7) Absolute contraindication for $1 / 3$-dose PDT or FA \\
\hline & 8) Myopia ( $\leq 6 \mathrm{D})$, hyperopia ( $>6 \mathrm{D}$ ) and aphakia \\
\hline
\end{tabular}

PDT, photodynamic therapy; CT, optic coherence tomography; CSC, central serous chorioretinopathy; CNV, choroidal neovascularization; LP, laser photocoagulation; TA, triamcinolone acetonede; VEGF, vascular endothelial growth factor; FA, fluorescein angiogramphy; ICGA, indocyanine green angiogramphy Photodynamic therapy. 
using spectral-domain OCT (Spectralis HRA+OCT, Heidelberg Engineering, Heidelberg, Germany). During post-PDT visits, the locations assessed at baseline were re-examined using the eye-tracking function of Spectralis OCT. OCT images were used to measure central foveal thickness (CFT), neuroretinal thickness (NRT), height of SRF, and subfoveal choroidal thickness (SCT). They were manually measured beneath the fovea on horizontal and vertical OCT images using the built-in-caliper function of the Heidelberg eye explore software. The average of the horizontal and vertical scan measurements was used in evaluations.

CFT was defined as the distance between the internal limiting membrane and the outer portion of the RPE. Height of SRT was defined as the distance between the outer surface of the sensory retina and the inner portion of the RPE.NRT was calculated by subtracting SRF height from CFT. SCT was defined as the distance between the outer portion of RPE and the inner surface of the sclera. Baseline FA and ICGA were performed using Spetralis HRA (Heidelberg Engineer, Heidelberg, Germany) in all patients. Window defect was defined as an area of hyperfluorescence on FA without an increase in size from early to late phase. All areas of the window defect on a 30-degree field of view were measured and summed up using the built-in caliper function of the Heidelberg Eye Explore software. Retreatment was considered if SRF persisted for over 1 year after PDT or if SRF recurred and persisted for 3 months. Auto fluorescence was used to check the RPE at baseline and after PDT.

4) Statistical comparisons were made between the baseline and 1, 2, 3, 6, 12 months after PDT using Student's t-test for numeric data with SPSS19.0 software (SPSS Inc., Chicago, Illinois, USA). A P-value $<0.05$ was considered statistically significant.

\section{Results}

59 eyes of 59 subacute CSC patients were included in this study. Among the patients, 38 were men and 21 were women with a mean age of $45.47 \pm 5.30$ (range, 34 - 48 years) at the time of 1/3-dose verteporfin PDT. The overall mean follow-up period was $14.1 \pm 8.4$ months (range 12 - 23 months). There was no eye with previous exposure to corticosteroids (25 50\% eyes of patients with a history of smoking). The mean PDT spot area was $14.2 \pm 9.1 \mathrm{~mm}^{2}$ (range, $3.4-4.2$ $\mathrm{mm}^{2}$ ). The mean history of CSC was $4.32 \pm 1.28$ months (rang, $3-6$ months).

After initial 1/3-dose PDT, absorption of SRF was complete in all (100\%) eyes; No eye had SRF recurrence 12 months after PDT.

1) Best-Corrected Visual Acuities

The mean LogMARBCVA at baseline and 1, 2, 3, 6 and 12 months after 1/3-dose PDT was $0.553 \pm 0.11,0.201 \pm 0.13,0.149 \pm 0.10,0.137 \pm 0.09,0.143 \pm$ 0.07 and $0.148 \pm 0.08$ respectively. Baseline BCVA significantly improved at 2, 3 and 6 months after PDT (all $\mathrm{P}<0.01$ ). The BCVA values at each time point were compared between the baseline and deferent follow-up time after 1/3-dose PDT (Figure 1). 


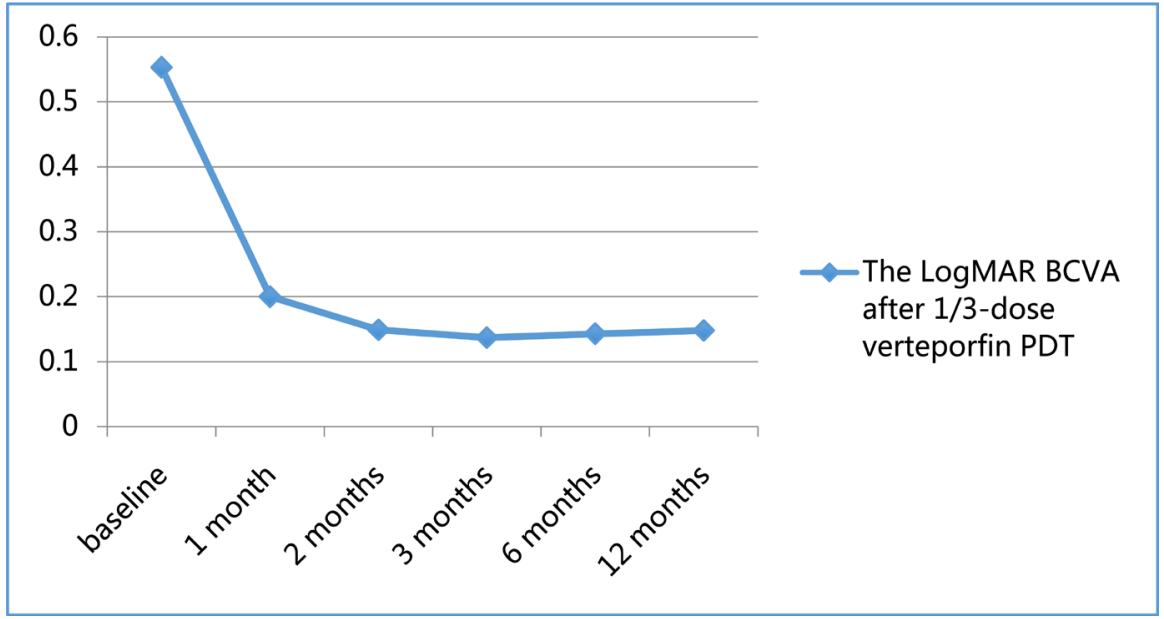

Figure 1. The LogMAR BCVA after 1/3-dose verteporfin PDT.

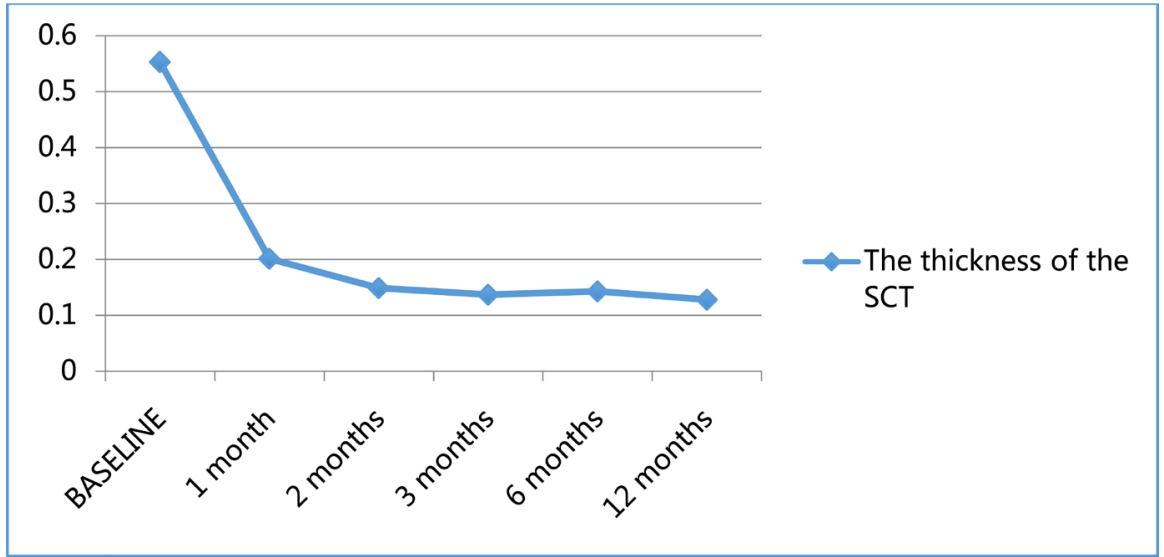

Figure 2. The thickness of the SCT after 1/3-dose verteporfin PDT.

\section{2) SCT Changes after 1/3-Dose Verteporfin PDT}

The mean SCT at baseline and at 1, 2, 3, 6 and 12 months after 1/3-dose PDT was $458.25 \pm 24.23 \mu \mathrm{m}, 379.31 \pm 9.31 \mu \mathrm{m}, 353.24 \pm 8.57 \mu \mathrm{m}, 349.35 \pm 7.51 \mu \mathrm{m}$, $365.23 \pm 8.08 \mu \mathrm{m}$, and $358.41 \pm 7.96$ respectively. After $1 / 3$-dose PDT, baseline SCT became significantly thinner at 1,2 , and 3 months (all $\mathrm{P}<0.01$ ). Baseline SCT was not significantly compared with 6 months $(P=0.41)$ (Figure 2$)$.

3) There was no CNV, atrophy of RPE and other side effect in all patients. Auto fluorescence had no change at baseline and after PDT.

\section{Discussion}

Our study suggested that relatively early PDT might improve outcomes of subacute CSC. Acute CSC was diagnosed as the history shorter than 3 - 4 months, and the chronic CSC was more than 6 months [1] [3]. But the period of more than 3 months but shorter than 6 months was not mentioned. In our study, the subacute CSC was diagnosed that the history was more than 3 months but shorter than 6 months. Accumulation of serous retinal detachment (SRD) results in damage of photoreceptor cells retinal atrophy [19] [20] and RPE damage [7]. 
These reports suggested that accumulation of SRD and aging may cause reduction of RPE function. Previous short-term and long-term studies showed a significant correlation between the baseline and final BCVA values [16] [22] [21]. In these studies, half-dose PDT was used to treat the chronic CSC. After 1 or 3 years follow-up, there were 33\% patients had recurrent SFD and lowly BCVA. Ooto et al. reported that damage of photoreceptor cells was associated with loss of BCVA [19]. Damage of photoreceptor cells may not be restored even after a prolonged period after half-dose PDT. A relatively early PDT before the occurrence of visual impairment is important to maintain good visual acuity in eyes with CSC. Our study showed that $1 / 3$-dose verteporfin PDT in subacute CSC can maintain or improve the BCVA.

Our study suggested that early 1/3-dose verteporfin PDT may actively improve outcomes of subacute CSC .Inoue et al. reported that eyes without intense hyperfluorescence on ICGA were not responsive to PDT and that the recurrence rate was predicted to be high [23]. This suggested that PDT was effective in resolution of SRD only in cases with high hydrostatic pressure in the choroid. They also suggested that in eyes with CSC and intermediate hyperfluorescence, a disrupted RPE might not tolerate small changes in choroidal hydrostatic pressure, resulting in recurrences. SRD accumulation would occur when hydrostatic pressure in the choroid exceeds RPE tolerance. PDT induces occlusion of the choriocapillaries [24] and reduces hydrostatic pressure in the choroid. As PDT does not restore disrupted RPE, it is not effective in cases with disrupted RPE and intermediate hydrostatic pressure. These findings and our study suggested the importance of relatively early treatment of PDT, before the disruption of RPE.

Our study suggested that the SCT were lower after PDT than baseline in all cases and the SRF were resulted. That means the 1/3-dose PDT is also effectively in subacute CSC. The previous study showed that half-dose PDT was effective with chronic CSC [16] [21]. There were also some reports showed that there were CNV and atrophy of RPE in chronic CSC with half-dose PDT. Although some cases were relative with long history of CSC and disrupted RPE, the halfdose of PDT may be also damage the choroidal vessel or RPE. 1/3-dose PDT had effect in chronic CSC in the previous reports [18]. Reduced dose of PDT (1/3-dose) was used in our study. It suggested that 1/3-dose PDT was effectively and safely with subacute CSC. There was no any CNV and atrophy of RPE in all cases. There was no any safe event during follow-up.

The choroid of the eyes with CSC is thicker than that of normal eyes [25]. Previous studies have shown that the choroidal thickness rapidly decreased after successful PDT and that it remained decreased for years [8] [26] [27] [28]. The previous study reported that choroidal thickness decreased by $4.1 \mu \mathrm{m}$ for every year increase in age [29]. Therefore, gradual decrease of choroidal thickness may be caused by an aging effect, during which the effect of the PDT may no longer exist. To the best of our knowledge, our study was the first to demonstrate the effect of 1/3-dose PDT in subacute CSC and the changes in choroidal thickness 
of eyes in the long-term. Further study is needed to find the long-term changes in choroidal thickness and other images after PDT.

Our study had several limitations, including its nonrandomized and long-term retrospective. Some patients may have a long history of CSC and did not remember the exact duration of symptoms even though these were our exclusion ceria.

\section{Conclusion}

1/3-dose PDT for subacute CSC had high anatomical and functional success rates in the 12 months follow-up. Relatively early 1/3-dose PDT is recommended in order to maintain good visual function of patients with subacute CSC. 1/3-dose PDT was safe and effective in subacute CSC.

\section{Acknowledgements}

\section{Supporting information}

Shen Yang scientific and technical unit

\section{Author Contributions}

Each author, including Li Xu, Qiushi Liu, Xiaotong Zhuang, Dongning Liu had contributed to the design, collection, analysis, interpretation of data, manuscript writing.

\section{References}

[1] Gass, J.D. (1967) Pathogenesis of Disciform Detachment of the Neuroepithelium. American Journal of Ophthalmology, 63, 1-139.

[2] Yannuzzi, L.A., Slakter, J.S., Gross, N.E., Spaide, R.F., Costa, D., Huang, S.J., et al. (2003) Indocyanine Green Angiography Guided Photodynamic Therapy for Treatment of Chronic Central Serous Chorioretinopathy: A Pilot Study. Retina, 23 288-298. https://doi.org/10.1097/00006982-200306000-00002

[3] Jeremy, M. and Thomas, R. (2008) Central Serous Chorioretinopathy. American Journal of Optometry, 79, 241-251.

[4] Prunte, C. and Flammer, J. (1996) Choroidal Capillary and Venous Congestion in Central Serous Chorioretinopathy. American Journal of Ophthalmology, 121, 26-34. https://doi.org/10.1016/S0002-9394(14)70531-8

[5] Klein, M.L., Van Buskirk, E.M., Friedman, E., Gragoudas, E. and Chandra, S. (1974) Experience with Nontreatment of Central Serous Choroidopathy. Archives of Ophthalmology, 91, 247-250.

[6] Gilbert, C.M., Owens, S.L., Smith, P.D. and Fine, S.L. (1984) Long-Term Follow-Up of Central Serous Chorioretinopathy. British Journal of Ophthalmology, 68, 815-820. https://doi.org/10.1136/bjo.68.11.815

[7] Imamura, Y., Fujiwara, T. and Spaide, R.F. (2011) Fundus Auto Fluorescence and Visual Acuity in Central Serous Chorioretinopathy. Ophthalmology, 118, 700-705.

[8] Chan, W.M., Lam, D.S., Lai, T.Y., Tam, B.S., Liu, D.T. and Chan, C.K. (2003) Choroidal Vascular Remodeling in Central Serous Chorioretinopathy after Indocyanine Green Guided Photodynamic Therapy with Verteporfin: A Novel Treatment at the Primary Disease Level. British Journal of Ophthalmology, 87, 1453-1458. 
[9] Cardillo Piccolino, F., Eandi, C.M., Ventre, L., Rigault de la Longrais, R.C. and Grignolo, F.M. (2003) Photodynamic Therapy for Chronic Central Serous Chorioretinopathy. Retina, 23, 752-763.

[10] Colucciello, M. (2006) Choroidal Neovascularization Complicating Photodynamic Therapy for Central Serous Retinopathy. Retina, 26, 239-242. https://doi.org/10.1097/00006982-200602000-00027

[11] Lee, P.Y., Kim, K.S. and Lee, W.K. (2009) Severe Choroidal Ischemia following Photodynamic Therapy for Pigment Epithelial Detachment and Chronic Central Serous Chorioretinopathy. Japanese Journal of Ophthalmology, 53, 52-56. https://doi.org/10.1007/s10384-008-0613-Z

[12] Lai, T.Y., Chan, W.M., Li, H., Lai, R.Y., Liu, D.T. and Lam, D.S. (2006) Safety Enhanced Photodynamic Therapy with Half Dose Verteporfin for Chronic Central Serous Chorioretinopathy: A Short-Term Pilot Study. British Journal of Ophthalmology, 90, 869-874. https://doi.org/10.1136/bjo.2006.090282

[13] Chan, W.M., Lai, T.Y., Lai, R.Y., Liu, D.T. and Lam, D.S. (2008) Half-Dose Verteporfin Photodynamic Therapy for Acute Central Serous Chorioretinopathy: One-Year Results of a Randomized Controlled Trial. Ophthalmology, 115, 1756-1765.

[14] Karakus, S.H., Basarir, B., Pinarci, E.Y., Kirandi, E.U. and Demirok, A. (2013) Long-Term Results of Half-Dose Photodynamic Therapy for Chronic Central Serous Chorioretinopathy with Contrast Sensitivity Changes. Eye, 27, 612-620. https://doi.org/10.1038/eye.2013.24

[15] Tseng, C.C. and Chen, S.N. (2015) Long-Term Efficacy of Half-Dose Photodynamic Therapy on Chronic Central Serous Chorioretinopathy. British Journal of Ophthalmology, 99, 1070-1077. https://doi.org/10.1136/bjophthalmol-2014-305353

[16] Fujita, K., Imamura, Y., Shinoda, K., Matsumoto, C.S., Mizutani, Y., Hashizume, K., et al. (2015) One-Year Outcomes with Half-Dose Verteporfin Photodynamic Therapy for Chronic Central Serous Chorioretinopathy. Ophthalmology, 122, 555-561.

[17] Chan, W.M., Lai, T.Y., Lai, R.Y., Tang, E.W., Liu, D.T. and Lam, D.S. (2008) Safety Enhanced Photodynamic Therapy for Chronic Central Serous Chorioretinopathy: One-Year Results of a Prospective Study. Retina, 28, 85-93. https://doi.org/10.1097/IAE.0b013e318156777f

[18] Liu, D.-N., Xu, L. and Gao, G.-J. (2012) Efficacy of Photodynamic Therapy with 1/3 Dose Verteporfin for Treating Acute Central Serous Chorioretinopathy. Journal of Ophthalmology, 12, 709-710.

[19] Ooto, S., Hangai, M., Sakamoto, A., Tsujikawa, A., Yamashiro, K., Ojima, Y., et al. (2010) High-Resolution Imaging of Resolved Central Serous Chorioretinopathy using Adaptive Optics Scanning Laser Ophthalmoscopy. Ophthalmology, 117, 1800-9.9e1-2.

[20] Wang, M.S., Sander, B. and Larsen, M. (2002) Retinal Atrophy in Idiopathic Central Serous Chorioretinopathy. American Journal of Ophthalmology, 133, 787-793.

[21] Lim, J.I., Glassman, A.R., Aiello, L.P., Chakravarthy, U., Flaxel, C.J., Spaide, R.F., et al. (2014) Collaborative Retrospective Macula Society Study of Photodynamic Therapy for Chronic Central Serous Chorioretinopathy. Ophthalmology, 121, 1073-1078.

[22] Fujita, K., Imamura, Y., Shinoda, K., et al. (2015) One-Year Outcomes with Half-Dose Verteporfin Photodynamic Therapy for Chronic Central Serous Chorioretinopathy. American Journal of Ophthalmology, 122, 555-561.

[23] Inoue, R., Sawa, M., Tsujikawa, M. and Gomi, F. (2010) Association between the Ef- 
ficacy of Photodynamic Therapy and Indocyanine Green Angiography Findings for Central Serous Chorioretinopathy. American Journal of Ophthalmology, 149, 441-6.e1-2.

[24] Schlotzer-Schrehardt, U., Viestenz, A., Naumann, G.O., Laqua, H., Michels, S. and Schmidt-Erfurth, U. (2002) Dose Related Structural Effects of Photodynamic Therapy on Choroidal and Retinal Structures of Human Eyes. Graefe's Archive for Clinical and Experimental Ophthalmology, 240, 748-757.

https://doi.org/10.1007/s00417-002-0517-4

[25] Imamura, Y., Fujiwara, T., Margolis, R. and Spaide, R.F. (2009) Enhanced Depth Imaging Optical Coherence Tomography of the Choroid in Central Serous Chorioretinopathy. Retina, 29, 1469-1473. https://doi.org/10.1097/IAE.0b013e3181be0a83

[26] Pryds, A. and Larsen, M. (2012) Choroidal Thickness Following Extrafoveal Photodynamic Treatment with Verteporfin in Patients with Central Serous Chorioretinopathy. Acta Ophthalmologica, 90, 738-743.

https://doi.org/10.1111/j.1755-3768.2011.02157.x

[27] Maruko, I., Iida, T., Sugano, Y., Furuta, M. and Sekiryu, T. (2011) One-Year Choroidal Thickness Results after Photodynamic Therapy for Central Serous Chorioretinopathy. Retina, 31, 1921-1927. https://doi.org/10.1097/IAE.0b013e31822bf6b1

[28] Maruko, I., Iida, T., Sugano, Y., Ojima, A., Ogasawara, M. and Spaide, R.F. (2010) Subfoveal Choroidal Thickness after Treatment of Central Serous Chorioretinopathy. Ophthalmology, 117, 1792-1799.

[29] Wei, W.B., Xu, L., Jonas, J.B., Shao, L., Du, K.F., Wang, S., et al. (2013) Subfoveal Choroidal Thickness: The Beijing Eye Study. Ophthalmology, 120, 175-180. 